\title{
Batería Neuropsicológica Luria Inicial y procesos atencionales
}

\author{
Luria's Initial Neuropsychological Battery and atenttional process
}

\author{
Yaser Ramírez-Benitez ${ }^{1 *}$, Miriela Diaz-Bringas ${ }^{1}$, Rosário Som aboano ${ }^{1}$, Daime Hernando-Cuba ${ }^{1}$ \\ 1 Servicio de Neuropsicología. Centro Docente de Rehabilitación del Neurodesarrollo Rosa Luxemburgo. Cárdenas. Cuba.
}

\section{Resumen}

Introducción: El TDAH es una de las alteraciones del neurodesarrollo caracterizada por presentar síntomas nucleares: inatención, hiperactividad e impulsividad y por sus comorbilidades. Por ello, se necesitan instrumentos neuropsicológicos que identifiquen todos los sistemas cognitivos en desarrollo. La batería Luria Inicial (BLI) se creó con el objetivo de identificar y seguir los niños con alteraciones neuropsicológicas. Material-Método: La BLI tiene la debilidad de identificar directamente los problemas atencionales. Se utilizó como complementario las pruebas de atención sostenida visual/auditiva para evaluar la atención orientación y la tarea de interferencia perceptual para la atención ejecución. La muestra fue de 29 niños de 6 años de edad que habían sido identificados desde el primer año de vida con signos de hiperactividad. La correlación fue realizada por una prueba de regresión lineal en busca de debilidades y fortalezas discriminativas. Resultados: La correlación demostró ser más fuerte en los subtest de las funciones ejecutivas de la BLI. Los niños mostraron debilidad en la motricidad manual, orientación espacial, discriminación fonemática y en semejanzas-diferencias. De la muestra,15 presentaron problemas en la atención orientación auditiva y 9 en la visual. 20 niños presentaron dificultades en la atención ejecutiva. La tarea de interferencia perceptual resultó ser una importante herramienta para evaluar la capacidad de inhibición. Conclusiones: Las pruebas de atención orientación y ejecutivas utilizadas son consistentes para evaluar la atención como complemento a la BLI. Los primeros signos de hiperactividad predicen problemas en la atención ejecutiva en el desarrollo.

Palabras clave: atención, neurodesarrollo, batería Luria Inicial

\begin{abstract}
Introduction: ADHD is a neurodevelopmental disorders are characterized by core symptoms: inattention, hyperactivity and impulsivity and comorbidities. That is why neuropsychological tools are needed to identify all developing cognitive systems. Initial Luria Battery (BLI) was created with the aim of identifying and tracking children with neuropsychological disorders. MaterialMethod: The BLI has directly identified the weakness of attentional problems. Was used as complementary tests of sustained attention visual / hearing to assess the care orientation and perceptual interference task execution for attention. The sample included 29 children under 6 years of age who had been identified since the first year of life with signs of hyperactivity. The correlation was performed by a linear regression test for weaknesses and strengths discriminative. Results: The correlation proved to be stronger in the subtest of executive functions of the BLI. The children showed motor weakness in the manual, spatial orientation, discrimination and phonemic similarities - differences. Sample 15 had attention problems auditory orientation and 9 in the visual. 20 children had difficulties in executive attention. The perceptual interference task proved to be an important tool to assess the ability of inhibition. Conclusions: The tests of attention and executive guidance are consistently used to assess attention in complementarity to the BLI. The first signs of hyperactivity, attention problems predict the development executive.
\end{abstract}

Keywords: attention, neurodevelopment, battery Luria Inicial

\footnotetext{
"Correspondencia: neurocar.mtz@infomed.sld.cu. Centro Docente de Rehabilitación del Neurodesarrollo Rosa Luxemburgo. Cárdenas, Calle Real, Cuba. Teléfono: +53 (45) 525 232, +53 (45) 525233

Recibido: 12-10-10. Revisión desde: 19-10-10. Aceptado: 19-05-11
}

DOI: $10.5839 / \mathrm{rcnp} .2011 .0601 .01$

Copyright $\odot 2011$ Universidad de La Frontera ISSN 0718-4913 (versión en línea) 


\section{Introducción}

La batería neuropsicológica Luria Inicial (BLI) (Ramos-Manga, 2006) para niños entre 4 a 6 años, se creó con el objetivo de identificar y seguir las alteraciones neuropsicológicas en la edad preescolar, y determinar las características preacadémicas del niño antes de comenzar la escolarización.

El instrumento fue bien recibido y validado en México, en la Universidad de Potosí (Bausela, 2008). En Cuba se recibió el mismo año que comenzó a comercializarse y tuvo un impacto terapéutico e investigativo de gran interés en el grupo de trabajo del departamento de endocrinología del Hospital Pediátrico de Cienfuegos (Ramírez, 2008; Ramírez-B, Marchena, 2008a; Ramírez, Marchena, 2009) y en el Departamento de Neuropsicología del Centro de Rehabilitación de Neurodesarrollo en Cárdenas, Matanzas.

El Departamento de Neuropsicología del Centro de Neurodesarrollo tiene la impronta de diagnosticar, seguir y crear estrategias de rehabilitación cognitivas en los diferentes problemas del neurodesarrollo, como el TDAH, trastornos del aprendizaje específicos (dislexia-discalculia) e inespecíficos, y trastornos de espectro autista, entre otros, alteraciones que predominantemente pueden ser identificadas por marcadores cognitivos conductuales (Demont \& Gombert, 2004; Alegría \& Mousty, 2004; Ramus et al., 2003).

El TDAH es una de las alteraciones del neurodesarrollo que ha sido más diagnosticada y estudiada en las últimas décadas. EI DSM-IV considera que es una alteración caracterizada por tres síntomas nucleares: inatención, hiperactividad e impulsividad (Colectivo de autores, 1994).

Bien se conoce que la comorbilidad es una parte importante en todas las alteraciones del neurodesarrollo, incluyendo el TDAH. Esta característica es referida no solo por las investigaciones cognitivas conductuales, también los estudios genéticos defienden la heterogeneidad en la expresión del fenotipo. Se estima que la heredabilidad media del cuadro sea 0,77 , superior a la esquizofrenia $(0,75)$, siendo la más heredable de las alteraciones mentales (Vaquerizo, 2008).

El fenotipo cognitivo del TDAH se caracteriza por los tres síntomas nucleares anteriormente mencionados, pero es habitual que el TDAH curse acompañado de trastornos de conductas, dificultades emocionales, fracaso escolar o dificultades de aprendizaje (Portellano, 2007). También, estos niños presentan signos neurológicos menores, como alteraciones viso-perceptivas, déficits psicomotores o cuadros apráxicos (Portellano, 2007).

El departamento tiene la obligación de discriminar y reconocer el fenotipo cognitivo del TDAH en los niños desde los primeros años de nacidos. El protocolo que ha seguido el centro desde su fundación ha permitido identificar y seguir los niños con/sin TDAH (Bringas, 2009).

$\mathrm{Si}$ en los primeros años de la etapa temprana se pueden apreciar signos asociados al cuadro, al final de la etapa se puede percibir con mayor claridad los síntomas nucleares y sus comorbilidades. Por ello, surge la necesidad de utilizar instrumentos que exploren todos los sistemas en desarrollo.

En lengua castellana existe dos instrumentos neuropsicológicos que ha sido de mucho valor investigativo y terapéutico: el CUMANIN de Portellano, 2000, y la BLI de Ramos-Manga, 2006. Ambos permiten identificar el cuadro de TDAH y las demás alteraciones acompañantes. Actualmente, el instrumento BLI es una prueba obligatoria en el protocolo de pruebas neuropsicológicas del niño preescolar diagnosticado y seguido en el Centro de Rehabilitación del Neurodesarrollo de Cárdenas.

La BLI tiene la fortaleza de evaluar y seguir el curso del desarrollo de las funciones ejecutivas desde los avances en las habilidades motoras del niño, las funciones lingüísticas alcanzadas, los procesos de memoria a corto plazo en dos modalidades, la rapidez de procesamiento y el proceso de lateralización como medida urgente de la especialización hemisféricas que cursa en esta etapa de la vida; sin embargo, su debilidad es no evaluar directamente los procesos atencionales. En la bibliografía no existen estudios que justifiquen este último planteamiento, lo cual es una razón para realizar este estudio.

El impacto de la batería para discriminar los signos nucleares y secundarios del TDAH ha sido determinado en (Ramos-Manga, 2006) y, recientemente, en un estudio de caso (Bausela y Ramírez, 2010). Pero aun así queda con objetividad esta debilidad.

El subtest 4 de la BLI, Regulación verbal del acto motor, sustentado en el principio go-no go de Luria, es considerado como altamente discriminativo para determinar la impulsividad y la hiperactividad, pero no es un subtest que mida la atención de los participantes, sino que permite inferir el estado de los procesos atencionales.

Los procesos atencionales, según Posner (2005), presentan tres dominios (Atención Alerta, Orientación y Ejecutiva), que no comparten ni las bases neurales, ni los neurotransmisores, ni la misma expresión genética (Fan, Posner y Fonsella, 2002).

Abordar la problemática de que la batería Luria Inicial no presenta fortalezas para evaluar los procesos atencionales es un punto que puede ser tratado con instrumentos complementarios que evalúen la atención según el modelo de Posner (2005).

Para ello, consideramos que el uso de las pruebas de atención sostenida visual y auditiva de Hernández (2008) puede ser de gran utilidad en la investigación de la Atención Orientación en el niño preescolar.

En el caso del dominio ejecutivo, el uso de la tarea de interferencia perceptual (paradigma stroop) (sonidos-imagen) puede ser efectiva para evaluar la atención ejecutiva en el niño preescolar (Montgomery et al., 2008; Witall et al., 2005).

\section{Material y Método}

En el Centro de Rehabilitación del Neurodesarrollo de Cárdenas, Matanzas, se seleccionó a 29 niños con 6 años de edad que habían sido seguidos desde el primer año de nacidos por presentar alguna alteración en su neurodesarrollo.

Se aplicó la BLI a los niños para obtener el perfil neuropsicológico en la edad $y$, como complementario, las pruebas de atención sostenida visual y auditiva (Hernández, 2008), para obtener Atención Orientación, y la prueba de Tareas Interferencia Perceptual, para obtener Atención Ejecución. Una vez con los datos, se aplicó la regresión lineal paso a paso entre los subcomponentes de las pruebas de atención y los subtest de la batería. 


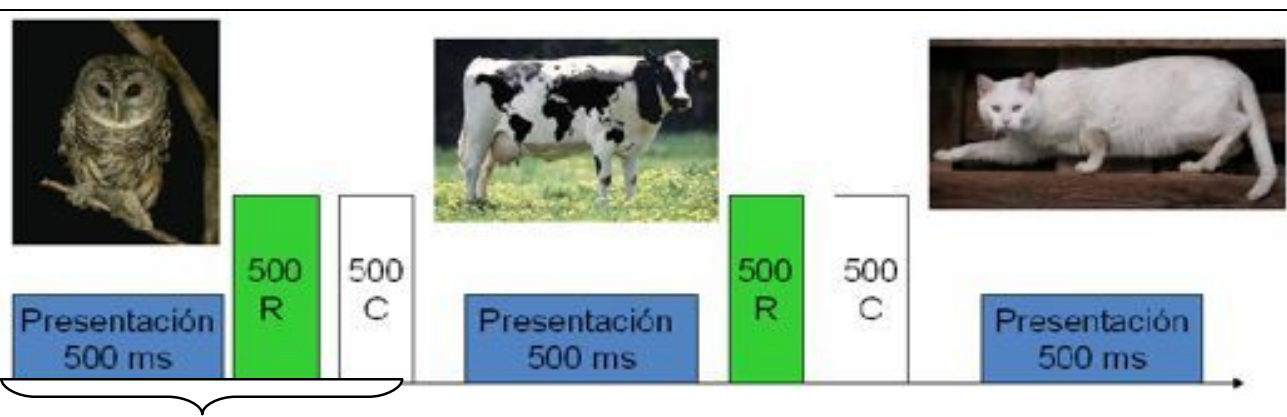

SOA - $1500 \mathrm{~ms}$ (sonidos-figuras)

Figura 1. Carta de presentación de la tarea de Interferencia Perceptual.

La Tarea de Interferencia Perceptual (TIP) consiste en simular un efecto stroop con sonidos e imágenes de animales conocidos universalmente por los niños. La primera parte consiste en reconocer los sonidos del animal y enseñarle su figura (no evaluativo), para luego indicarle la consigna de la prueba (evaluativo).

Consigna: "Vas a escuchar sonidos de animales, pero, a la vez, vas a ver figuras de animales. A veces los sonidos coinciden con el animal, pero a veces no. Debes estar bien atento. Tiene que seleccionar siempre los sonidos del caballo, aun cuando la figura no sea la del caballo".

Diseño: La prueba consta de 300 sonidos de animales (búfalo, búho, caballo, cabra, cerdo, cotorra, coyote, elefante, gallo, gato, hiena, hipopótamo, jabalí, mono, oso, pájaro, pato, perro, oveja y vaca).

Duración: En 5 minutos el niño debe identificar 5 dianas por minutos para constituir 25 dianas en todo la prueba. El SOA (stimulus outcome asyncrony) es de $1.500 \mathrm{~ms}$ (500 ms presentación del estímulo- $500 \mathrm{~ms}$ respuesta verbal- $500 \mathrm{~ms}$ cambio de estímulo).

Estímulo diana: relinchos del caballo.

\section{Resultados}

La regresión lineal evidencia un coeficiente de determinación significativo $\left(p>0,05\right.$, beta $(0,324) \quad r^{2}(0,72)$ entre los subcomponentes de los instrumentos de la atención y los subtest de la BLI. El coeficiente $r^{2}$ tiene relación directa con el coeficiente de correlación de Pearson y un valor estadístico de causalidad. Los resultados demostraron que los subtest de las funciones ejecutivas tienen mayor correlación que los demás dominios de la batería, pero de manera indiferente en los subcomponentes de la atención (ver tabla 1).

Los modelos ANOVA factor único más significativos que se realizaron con las funciones ejecutivas se comportaron: Modelo Atención visual - funciones ejecutivas ( $F-1,306$, sign $(0,850)$ (Omisiones), Modelo Atención auditiva - funciones ejecutivas (F-1,834, sign $(0,524)$ (Acierto) y el Modelo Atención interferencia (F-1,205, sign $(0,892)$ (Comisiones).

Las correlaciones de las tareas de rapidez de procesamiento con las pruebas de atención indican la importancia de los procesos atencionales en el aprendizaje. Las tareas de rapidez de Denckla (1976) suelen ser buenas predictoras de los problemas de aprendizaje. La atencion visual correlacionó con las comisiones (colores, 0,951: dibujo, 0,638). La atencion auditiva y la atención interferencia correlacionaron con las omisiones (auditiva: colores, 0,653, dibujo, 0,638; interferencia: colores, 0,705; dibujo, 0,997).

Las correlaciones con la memoria en sus dos modalidades también indican la importancia de dosis atencionales para adquirir contenidos. No hay duda de que las bases neuronales implicadas en la memoria son fundamentales para el aprendizaje.

Otra importante contribución son las mediciones de la esterognosia (reconocimiento de objetos por el tacto) como buenas medidas del estado neurobiológico de los lóbulos parietales y su contribución en los procesos atencionales. EI lóbulo parietal izquierdo suele ser más sensible a los estímulos atencionales usados (Atencion visual: Omisiones (0,873); Atención auditiva: Aciertos $(0,864)$; Atencion interferencia: Omisiones $(0,814)$.

El perfil neuropsicológico demuestra que los puntos débiles en las funciones ejecutivas están en las habilidades visoespaciales (test 5) y en la motricidad manual (test 1 ).

En las funciones lingüísticas, los puntos débiles están en la discriminación de fonemas y en las habilidades de razonamiento verbal (test 9).

En la Atención orientación suelen ser más discriminativas las pruebas de atención audición que las visuales. Las omisiones y los aciertos tienen una mayor correlación con los problemas atencionales que con la hiperactividad. Las comisiones son importantes en la prueba de interferencia y tienen una mayor correlación con las dificultades por impulsividad / hiperactividad.

La tarea de interferencia perceptual correlaciona positivamente (comisiones) con los subtest que evalúan el desarrollo de motricidad del niño (regiones motoras primarias y premotoras), (motricidad manual $(0,816)$ y Gestos y Praxis $(0,758)$, los procesos de inhibición (orbito frontal/cíngulo), con Regulación verbal $(0,797)$ y con la esterognosia izquierda $(0,960)$ (parietal derecho), siendo una tarea importante para conocer el estado de funcionamiento de las regiones anteriores del encéfalo en desarrollo y su conectividad con regiones posteriores.

\section{Discusión}

El estudio correlacional demuestra tres importantes resultados con relación al desarrollo motor del niño. El dominio ejecutivo de la BLI es un excelente dominio para evaluar la atención, pero resulta ser menos específico. Una buena rehabilitación cognitiva exige un diagnóstico preciso demostrado por instrumentos específicos. 
Ramírez-Benitez, et al. Rev. chil. neuropsicol. 6(1): 1-6, 2011

\begin{tabular}{|c|c|c|c|c|c|c|c|c|c|}
\hline \multirow[t]{2}{*}{ Variable independiente } & \multicolumn{3}{|c|}{ Atención visual } & \multicolumn{3}{|c|}{ Atención auditiva } & \multicolumn{3}{|c|}{ Atención interferencia } \\
\hline & $\mathrm{A}$ & 0 & $\mathrm{C}$ & $A$ & 0 & $\mathrm{C}$ & $A$ & 0 & $\mathrm{C}$ \\
\hline Motricidad manual & & 659 & & & ,816 & & & & 816 \\
\hline Orientación D-I & & ,503 & & & ,284 & & & & ,362 \\
\hline Gestos y praxis & & 677 & & & 147 & & & & ,758 \\
\hline Regulación verbal & & ,946 & & & ,634 & & & & 797 \\
\hline Orientación espacial & & ,953 & & & 147 & & & & ,591 \\
\hline Nombrar objetos & & & & & ,597 & & & & \\
\hline Audición fonemática & & & & & ,371 & & & & \\
\hline Vocabulario & & & & &, 574 & & & & \\
\hline Analogías & & & & & ,737 & & & & \\
\hline Cálculos sencillos & & & & &, 547 & & & & \\
\hline Rapidez de dibujos & & ,638 & ,850 & 771 & ,638 & & 997 & 997 & ,214 \\
\hline Rapidez de colores & & 951 & 927 & ,581 & ,653 & & 691 & ,705 & 870 \\
\hline Memoria verbal & & ,702 & 388 & ,849 & ,369 & & ,253 & ,265 & ,345 \\
\hline Memoria visual & & 764 & ,642 & 890 & ,464 & & ,478 & ,484 & ,546 \\
\hline Esterognosia izquierda & & ,354 & 352 & ,470 & ,259 & & 396 & ,386 & 960 \\
\hline Esterognosia derecha & & 873 & 120 & ,864 & ,487 & & ,796 & ,814 & ,278 \\
\hline
\end{tabular}

Tabla 1. Regresión lineal de los subtest de la BLI y los subcomponentes de las pruebas de atención al 95\%.

Valores Modelos ANOVA factor único.
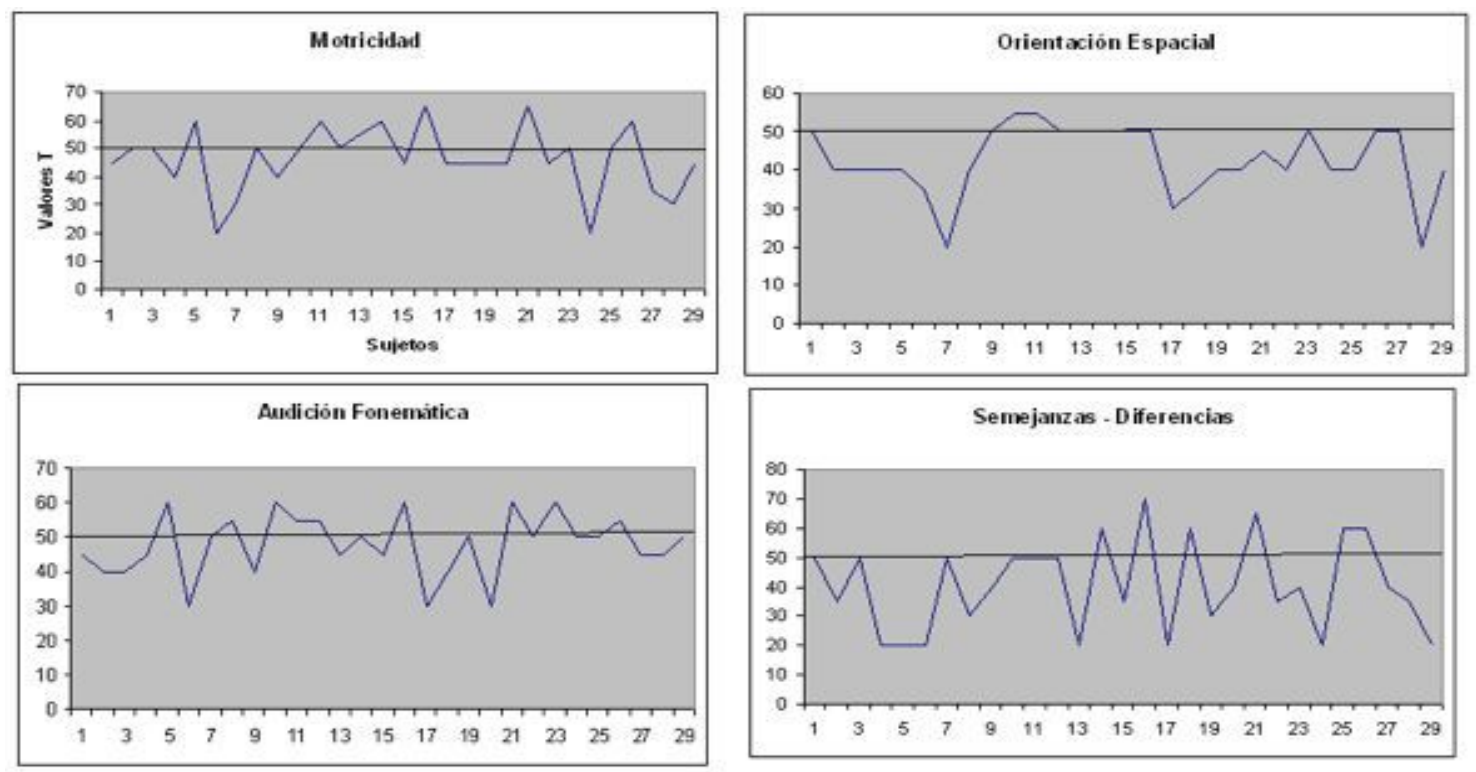

Gráfico 2. Puntos débiles en el perfil neuropsicológico. Funciones ejecutiva: motricidad manual y orientación espacial. Funciones lingüísticas: Audición fonemática y semejanzas - diferencias. 


\section{Atención Orientación}

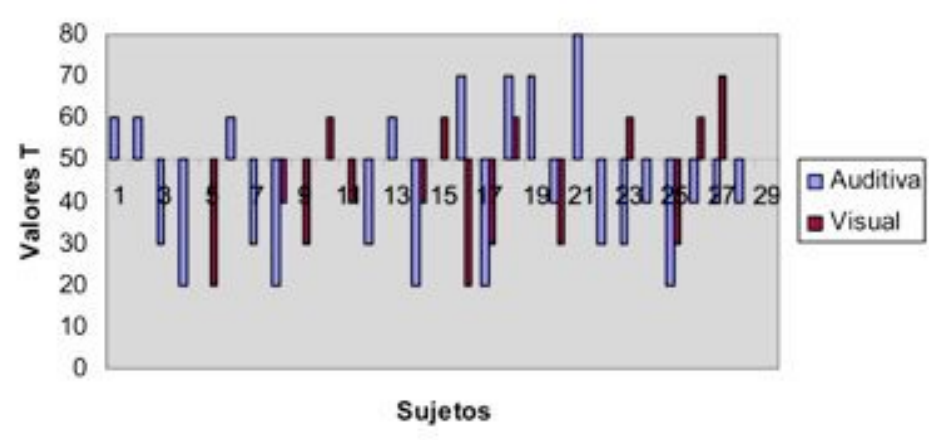

Gráfico 3. Valores T de los aciertos en las prueba Atención auditiva y Visual.

La atención ejecutiva puede ser evaluada con el dominio ejecutivo de la batería sin la utilización de la Tarea de Interferencia Perceptual $u$ otro instrumento que evalúe la atención. Las dificultades en los subtest 1,3 y 4 del dominio ejecutivo muestran el estado funcional de los procesos motores, pero a la vez expresan el desempeño de la atención ejecutiva. El desarrollo motor es fundamental para dar pronósticos de la atención. Los demás subtest ( 2 y 5 ) exigen una dosis atencional importante, pero evalúan más las habilidades espaciales que la atención. La rehabilitación motora del niño de 0 a 5 años puede ser un fuerte indicador para potenciar las vías neurobiológicas de la atención ejecutiva.

El hecho de que se habiliten los procesos motores complejos en el niño preescolar estimula las vías anteriores de la atención $y$, también, las vías posteriores. La motricidad requiere de dosis altas de atención, pero también de una ubicación espacial (conexiones fronto / parietales).

El funcionamiento motor del niño debe ser entrenado en la edad temprana, ya que de ello depende la fortaleza de conexiones sinápticas corticales y subcorticales de corta y larga distancia; además, permite potenciar las vías dopaminérgicas que tienen una representación cerebral precisamente en áreas implicadas con la motricidad.

Del entrenamiento motor se puede tener resultados cognitivos positivos en los últimos años de la etapa preescolar y principios de la escolar.

Una vez más se ha llegado a una generalización comentada por Luria en sus investigaciones en el siglo pasado con relación al desarrollo motor del niño; además, ha sido demostrada también por los autores de la batería Luria Inicial en este siglo.

Las principales comorbilidades apreciadas fueron en el funcionamiento lingüístico (discriminación fonemática) y en su capacidad oral para subordinar funciones cognitivas complejas en función del razonamiento verbal (ver gráfico 2). Ambas tienen una fuerte relación con problemas en el aprendizaje, ya sea de tipo específicos (dislexia-discalculia) o inespecíficos.

Las pruebas de Atención Orientación identificaron a 15 niños con problemas en la atención auditiva y 9 en la visual (ver gráfico 3). Estos resultados demuestran que la capacidad de los niños para seleccionar estímulos sensoriales está disminuida, ya sea por distracciones o por impulsos.
Las dos pruebas recogen aciertos, omisiones $y$ comisiones. Los dos primeros se han relacionado con la atención; por tanto, los bajos valores nos permitir decir que el niño ha disminuido su capacidad de selección por distracciones; en cambio, los altos valores en las comisiones se relacionan con los impulsos.

Las pruebas permiten analizar conductualmente si el nivel de selectividad de estímulos está disminuido por distracciones o por impulsividad.

Las distracciones, al parecer, tienen bases neurales asociadas a las vías neurobiológicas posteriores, ya que los aciertos y las omisiones tienen una mayor correlación con las pruebas de Atención Orientación.

Entre varios estímulos, el niño no es capaz de seleccionar uno, todos le resultan parecidos, pero los omite o los marca, hay una cierta inhibición.

Este nivel de selectividad también se encuentra limitado cuando los impulsos obligan al niño a ejecutar una respuesta. Al parecer, el control de la inhibición y la desinhibición permite dar respuesta a la tarea de interferencias, solo que los niños TDAH son más propensos a la desinhibición. La velocidad con que sucede la prueba y la confusión que ocasionan los estímulos activa unos complejos procesos de inhibición-desinhibición. Esto ocurre precisamente en regiones cerebrales tanto en adultos (Stelzel et al., 2009; Haupt et al., 2009) como en niños (Clément de Guibert et al., 2010), aparentemente predeterminado como un sistema de sobrevivencia.

En la muestra utilizada, solo 20 niños presentaron problemas en la capacidad de inhibición en la TIP, lo cual nos dice que el instrumento es capaz de identificar el déficit fundamental del TDAH.

Las tareas de interferencia son consideradas excelentes instrumentos para evaluar las funciones ejecutivas y se ha demostrado que activan regiones asociativas anteriores $y$ posteriores ante la ejecución de ellas.

$\mathrm{Si}$ las pruebas de Atención Orientación permiten conocer el estado neurobiológico de las vías atencionales posteriores, las pruebas de Interferencia nos ayudan a conocer cómo funcionan las dos vías atencionales en un proceso de selectividad. 
La investigación recomienda diseñar en el centro un programa de rehabilitación motora en niños con signos de hiperactividad desde el primer año, como vía para potenciar las bases neurales de la atención, para luego evaluarlos a los 5 ó 6 años. El centro ha trabajado hace mucho con esta rehabilitación, solo que no ha publicado resultados longitudinales.

\section{Conclusiones}

La BLI no evalúa elementos diagnósticos para conocer su estado. Los subtest 1, 3 y 4 del instrumento muestran el estado funcional de las principales redes neurobiológicas de la atención. Aun cuando estos subtest puedan darnos elementos diagnósticos, es preciso utilizar otros instrumentos complementarios para ser más específicos y rehabilitar el deficit. Los déficit en la prueba de atencion visual pueden orientar que las alteraciones de la red atencional son más significativas en las regiones posteriores del encéfalo, y los déficit en la atencion interferencia y auditiva puede orientar las alteraciones en las regiones temporo/frontales.

La rehabilitación del niño preescolar con TDAH exige que se apliquen ambos instrumentos para apreciar los déficits nucleares y comorbilidades. Los signos de hiperactividad desde el primer año de vida son signos predictores de problemas en la atención ejecutiva. Esta se puede rehabilitar con entrenamiento del funcionamiento motor en la etapa temprana del desarrollo.

\section{Agradecimientos}

Agradecimiento: La investigación ha sido realizada gracias a la colaboración de los doctores Francisco Ramos y Dionisio Manga.

\section{Referencias}

Alegria, J. \& Mousty, P. (2004). Les troubles phonologiques et métaphonologiques chez l'enfant dyslexique. Enfance, 3, 259-271.

American Psychiatric Association. Diagnostic and statistical manual of mental disorders (DSM-IV), 4 ed. Washington DC: APA, 1994.

Bausela-Herrera, E. (2008). Estudio de validación de la batería Luria Inicial en una muestra de escolares mexicanos. Revista Mexicana de Neurociencias, 9(2), 125-30.

Bausela-Herrera, E., Ramírez-Benítez, G., Martínez-Gutiérrez, OrozcoMoreno, C. I. (2010). Funcionamiento ejecutivo y Luria-Inicial: A propósito de un caso. Boletín de Pediatría, 50, 33-38.

Clément de Guibert, C., Maumet, C., Ferré, J. Ch., Jannin, P., Biraben, A., Allaire, C., Barillot, Ch., Rumeur, E. (2010). FMRI language mapping in children: A panel of language tasks using visual and auditory stimulation without reading or metalinguistic requirements. Neurolmage, 51, 897-909.

Demont, E. \& Gombert, J.E. (2004). L'apprentissage de la lecture: évolution des procédures et apprentissage implicite. Enfance, 3, 245-257.

Díaz-Bringas, M. (2009). Algoritmo de atención integral al trastorno de hiperactividad en edad temprana. Tesis Maestría. Matanzas, Cuba.

Fan, J., McCandliss, B.D, Flombaum, I., Fossella, J, Posner, M.I. (2005). The activacion of attentional networks. Neurolmage, 26, 471-479.

Fan J, McCandliss B.D., Sommer T, Raz M, Posner M.I. (2002). Testing the efficiency and independence of attentional networks. Journal of Cognitive Neuroscience, 3(14), 340-47.

Haupt et al. (2009). Activation of the caudal anterior cingulate cortex due to task-relate interference in an auditory Stroop paradigm. Human Brain Mapping, 30, 9.
Montgomery, D. E., Anderson, M., Uhl, E. (2008). Interference Control in Preschoolers: Factors Influencing Performance on the Day-Night Task. Infant and Child Develpment, 17, 457-470.

Pérez-Hernández, E. (2008). Desarrollo de los procesos atencionales. Tesis doctoral Universidad Complutense, Madrid, España. http://eprints.ucm.es/8447/

Portellano, J.A. (2007). Neuropsicología Infantil. Editorial Madrid, Síntesis.

Ramírez-Benítez. (2008). Signos neurológicos menores en la edad preescolar. Revista Mexicana de Neurociencias, 9(6), 445-453.

Ramírez-Benítez, Hussimy Marchena M. (2008a). Alteraciones neuropsicológicas en la hiperplasia adrenal congénita. Revista Cubana de Endocrinología, 19(3).

Ramírez-Benítez, Hussimy Marchena M. (2009). Características neuropsicológicas del niño preescolar con Hipotiroidismo Congénito en la Provincia de Cienfuegos. Revista Chilena de Neuropsicología, 4(1), 36-43.

Ramos, F., Manga, D. Luria Inicial: Evaluación neuropsicológica en la edad preescolar. Manual. TEA Ediciones, 2006.

Ramus, F., Rosen, S., Dakin, S. C., Day, B. L., Castellote, J. M., White, S. \& Firth, U. (2003). Theories of developmental dyslexia: Insights from a multiple case study of dyslexic adults. Brain, 126, 841-865.

Stelzel, C., Brandt, S.A., Schubert, T. (2009). Neural mechanisms of concurrent stimulus processing in dual tasks. Neurolmage, 48(1), 237-248.

Vaquerizo-Madrid, J. (2008). Evaluación clínica del trastorno por déficit de atención/hiperactividad, modelo de entrevista y controversias. Revista de Neurología, 46(1): S37-S4

Whitall, J., McMenamin, S., Getchell, N. (2005). Dual Motor Task Coordination in Children with and without Learning Disabilities. Adapted physical activity quaterly, 22(1), 21-38.

Denckla, M B, Rudel R G. (1976). Rapid automatized naming: Dyslexia differentiated from other learning disabilities. Neuropsychologia, $14,471-479$. 